\title{
Simulation of nonlinear instabilities in an attachment-line boundary layer
}

\author{
Ronald D. Joslin \\ NASA Langley Research Center, Hampton, Virginia 23681, U.S.A.
}

\begin{abstract}
The linear and the nonlinear stability of disturbances that propagate along the attachment line of a three-dimensional boundary layer is considered. The spatially evolving disturbances in the boundary layer are computed by direct numerical simulation (DNS) of the unsteady, incompressible Navier-Stokes equations. Disturbances are introduced either by forcing at the inflow or by applying suction and blowing at the wall. Quasi-parallel linear stability theory and a nonparallel theory yield notably different stability characteristics for disturbances near the critical Reynolds number; the DNS results confirm the latter theory. Previously, a weakly nonlinear theory and computations revealed a high wave-number region of subcritical disturbance growth. More recent computations have failed to achieve this subcritical growth. The present computational results indicate the presence of subcritically growing disturbances; the results support the weakly nonlinear theory. Furthermore, an explanation is provided for the previous theoretical and computational discrepancy. In addition, the present results demonstrate that steady suction can be used to stabilize disturbances that otherwise grow subcritically along the attachment line.
\end{abstract}

Running Title: Nonlinear Attachment-Line Instabilities

Dr. Ronald D. Joslin

Fluid Mechanics and Acoustics Division

NASA Langley Research Center

Mail Stop 170

Hampton, Virginia 23681-0001

(804)864-2234, Fax: (804)864-8801

E-mail: R.D.Joslin@larc.nasa.gov

Some results were originally presented as AIAA Paper No. 94-0826 at the AIAA 32nd Aerospace Sciences Meeting \& Exhibit, January 10-13, 1994, Reno, NV. 


\section{Introduction}

On a swept wing, many instability mechanisms occur that can lead to the catastrophic breakdown of laminar to turbulent flow. Contamination along the leading edge, TollmienSchlichting waves, stationary or traveling crossflow vortices, Taylor-Görtler vortices, or combinations of these modes are among the mechanisms that can lead to this breakdown. For brevity, the discussion here will be limited to disturbances in the region of the attachment line. For a more complete discussion of transition to turbulence on swept wings, refer to the work of Tuttle and Maddalon (1982), which includes a review of literature on laminar flow control, and that of Reed and Saric (1986), which includes a description of the known physical mechanisms associated with transition. Körner et al. (1987) present a German perspective on the laminarization of transport aircraft, and Gad-el-Hak and Bushnell (1991) discuss separation control on wings. The most recent and comprehensive overview of experiments, theory, and computations related to boundary-layer transition prediction and application to drag reduction is given by Arnal (1994).

Contamination at the leading edge results from turbulence at a fuselage/wing juncture, which travels out over the wing and contaminates otherwise laminar flow on the wing. If the Reynolds number of the attachment-line boundary layer is greater than some critical value, then this contamination inevitably leads to turbulent flow over the complete wing; this phenomenon has been demonstrated by Maddalon et al. (1990) and others. To correct this problem, Gaster (1965) placed a bump on the leading edge to prevent the turbulent attachment-line boundary layer from sweeping over the entire wing. This bump must be shaped to create a fresh stagnation point without generating a detrimental adverse pressure gradient. Outboard of the bump, a new laminar boundary layer forms.

Although the problem of turbulent flow that originates from the fuselage/wing juncture and contaminates the entire wing can be avoided by using a device such as the Gaster bump, a Reynolds number exists beyond which disturbances generated by surface im- 
perfections or particulates on the wing, when combined with noise, will eventually cause transition. If we assume that the initiated disturbances are sufficiently small, hydrodynamic stability theory could potentially be used to predict the spatial amplification and the decay of the disturbances along the attachment line. Gaster (1967) first examined this small-amplitude disturbance problem by using acoustic excitation along the attachment line of a swept cylinder model. Gaster fed the flow sine waves with various frequencies that were detected by a hot-film gauge on the attachment line. He noted that the recorded oscillations had preferred frequency bands that changed with tunnel speed and that this behavior was similar to that of traveling-wave instabilities. From his measurements, he concluded that the small-amplitude disturbances in an attachment-line boundary layer were stable for momentum-thickness Reynolds numbers $R_{\theta}$ below 170 (the critical Reynolds number was outside the experimental range); this value for the critical Reynolds number is close to the theoretical value of 200 , which is obtained by assuming a two-dimensional (2D) attachment-line boundary layer. Later, Cumpsty and Head (1969) experimentally studied large-amplitude disturbances and turbulent flow along the attachment line of a swept-wing model. They observed that laminar flow is stable to small-amplitude disturbances up to $R_{\theta} \simeq 245$ (which corresponds to the top speed of the tunnel). Cumpsty and Head note that this observation remains consistent with the theoretical value; an accurate theoretical value would need to account for three-dimensional (3D) effects. At the same time, Pfenninger and Bacon (1969) used a wing swept to $45^{\circ}$ to experimentally study the attachment-line instabilities in a wind tunnel that was capable of the larger speeds necessary to obtain unstable disturbances. With hot wires, they observed regular sinusoidal oscillations with frequencies comparable to the most unstable 2D modes of theory; these modes caused transition to occur at $R_{\theta} \simeq 240$. A continued interest in transition initiated near the attachment line of swept wings led Poll $(1979,1980)$ to conduct additional experiments. With the swept circular cylinder model of Cumpsty and Head (1969), Poll 
defined criteria for the onset of turbulence and identified the forms of the disturbances present in the flow. Like Pfenninger and Bacon (1969), Poll observed disturbances that amplified along the attachment line. He noted that no unstable modes were observed below $R_{\theta}=230$.

Using an eigenvalue problem approach, Hall et al. (1984) studied the linear stability of disturbances in the attachment-line boundary-layer flow called swept Hiemenz flow, which is illustrated in figure 1 . This $3 \mathrm{D}$ base flow is a similarity solution to the NavierStokes equations; hence, its use is advantageous in stability analyses. By assuming periodic instability modes along the attachment line, Hall et al. (1984) determined neutral curves with and without the presence of steady suction and demonstrated that the attachmentline boundary layer theoretically can be stabilized with small amounts of suction. Hereafter the Hall et al. (1984) approach is referred to as a nonparallel theory because the study accounted for all linear terms, including the wall-normal velocity component of the base flow. Figure 2 shows the agreement between the neutral curve of Hall et al. (1984) and the experimental results of Pfenninger and Bacon (1969) and Poll (1979, 1980). Spalart (1989) used a direct numerical simulation (DNS) approach, based on the fringe method, to study the leading-edge contamination problem. Small-amplitude disturbances were initialized with white noise. Both stable and unstable Reynolds-number test points were selected to assess the validity of the nonparallel theory by Hall et al. (1984). At the lower Reynolds number, all disturbances decayed; at the higher number, at least one mode was amplified. The critical number predicted by Hall et al. (1984) fell within the Reynolds number range used by Spalart; the results of the simulations indicate good qualitative agreement with the linear theory. Furthermore, Spalart (1989) demonstrated that classical Hiemenz flow is both linearly and nonlinearly stable. Theofilis (1993a) performed DNS of the 2D linear disturbances that propagate along the attachment line of swept Hiemenz flow. The DNS results agreed with the theory of Hall et al. (1984) near branch II of the neutral curve, but 
the DNS predicted growing modes in a region of theoretical decay near branch I. Theofilis (1993a) attributed the disagreement between DNS and theory near branch I of the neutral curve to the lack of DNS grid resolution; however, the DNS results differed by only a few percent from the theory. Small-amplitude computations of Jiménez et al. (1990) led to results that agreed with the linear results of Hall et al. (1984) for both branch I and branch II of the neutral curve. More recently, Joslin (1995) developed and used a fully 3D spatial DNS code that utilized a Chebyshev series in both the wall-normal and flow-acceleration directions and high-order finite and compact differencing in the attachment-line direction. The DNS results agreed with the neutral curve predicted by Hall et al. (1984). (Given the significant computational cost of the code described by Joslin (1995), a more efficient DNS code is used for the present study.)

As the initial amplitude of the disturbances in the attachment-line region become large, the experimental results show considerable discrepancy between the onset of transition and the linear critical Reynolds number. Pfenninger and Bacon (1969) placed a wire upstream of the attachment line and generated large-amplitude fluctuations in the boundary layer. They observed transition at $R_{\theta}=155$. In his study of leading-edge contamination, Pfenninger (1965) discovered through in-flight experiments that laminar flow could be obtained for $R_{\theta}<100$; for $R_{\theta}>100$, leading-edge contamination occurred. In their wind-tunnel experiments on a swept airfoil, Gregory and Love (1965) found that for $R_{\theta}>95$ complete turbulence occurred. Flight tests by Gaster (1967) showed that turbulent spots were first present at $R_{\theta}>88$. Cumpsty and Head (1967), and later Poll (1985), used a swept model in a wind tunnel to show that turbulence was damped for $R_{\theta}<99$ and that the leading edge was fully turbulent for $R_{\theta}>114$. Clearly, these experiments show that large roughness elements have an effect on the boundary layer that is similar to that of contamination. Namely, for $R_{\theta}<100$, disturbances are damped, and for $R_{\theta}>100$ the flow becomes turbulent (note the wide gap between the linear critical Reynolds number of 
$R_{\theta} \simeq 245$ and the nonlinear critical Reynolds number of $R_{\theta} \simeq 100$ ).

Hall and Malik (1986) strived to bridge the gap between the nonparallel linear theory and bypass transition Reynolds numbers by studying large-amplitude disturbances with weakly nonlinear theory and temporal DNS. They note that subcritical instability is observed at wave numbers that correspond to branch II of the neutral curve. Figure 2 shows the subcritical instability region of Hall and Malik (1986) with the nonparallel linear stability neutral curves. Hall and Malik note that no upper branch modes appear in the experimental results because of the subcritical nature of the bifurcation along most of the upper branch. Consistent with the experimental results, large-amplitude disturbances become unstable before the linear critical point and approach equilibrium states near branch I of the neutral curve. Both Jiménez et al. (1990) and Theofilis (1993b) failed to find this region of subcritical growth with a temporal DNS code. Jiménez et al. (1990) contend that this subcritical growth region does not exist.

Bridging the gap between the linear region of instability and the upstream region of bypass is important for transition prediction and control. As a first step toward this inherently nonlinear 3D process, the present study will focus on resolving the discrepancy between the weakly nonlinear theory and supporting computations of Hall and Malik (1986) and the two recent DNS computations. A well-tested 3D spatial DNS code described by Joslin, Streett, and Chang (1992) is used to independently study both the linear and nonlinear instabilities that initiate and develop along the attachment line of a swept Hiemenz flow. Regions near both branches (I and II) of the neutral curve are investigated with DNS to simultaneously verify the form of the disturbances used in the DNS and the nonparallel theory (eigenvalue approach) of Hall et al. (1984) for infinitesimal disturbances; to determine if regions of nonlinear instability growth can be found near branch II, which may resolve the discrepancy between the theory and the later DNS results; and to determine if steady suction can be used to control instability growth. 


\section{Problem formulation}

In general, the velocities $\underline{\tilde{u}}=(\tilde{u}, \tilde{v}, \tilde{w})$ and the pressure $\tilde{p}$ are solutions of the incompressible, unsteady Navier-Stokes equations. The instantaneous velocities $\underline{\tilde{u}}$ and the pressure $\tilde{p}$ may be decomposed into base and disturbance components as

$$
\underline{\tilde{u}}(\underline{x}, t)=\underline{U}(\underline{x})+\underline{u}(\underline{x}, t) \quad \text { and } \quad \tilde{p}(\underline{x}, t)=P(\underline{x})+p(\underline{x}, t)
$$

where the base flow is given by the velocities $\underline{U}=(U, V, W)$ and the pressure $P$, and the disturbance component is given by the velocities $\underline{u}=(u, v, w)$ and the pressure $p$. A Cartesian coordinate system $\underline{x}=(x, y, z)$ is used in which $x$ is aligned with the attachment line, $y$ is wall normal, and $z$ corresponds to the direction of flow acceleration away from the attachment line.

Originally described by Hall et al. (1984), the base flow referred to as a swept Hiemenz flow is a similarity solution to the incompressible 3D Navier-Stokes equations. Shown in figure 1 , the fluid comes straight down toward the wall; it turns away from the attachment line into the $\pm z$ directions to form a boundary layer. In the $x$ direction, the flow is uniform. In the absence of sweep, $U_{o}$ is equal to 0 and the flow reduces to the $2 \mathrm{D}$ stagnation flow first described by Hiemenz (1911). A boundary-layer thickness is defined in the $y z$-plane as $\delta=\sqrt{\nu L / W_{o}}$; a Reynolds number, as $R=U_{o} \delta / \nu$; and a transpiration constant, as $\kappa=V_{o} \sqrt{L / \nu W_{o}}$, where $\kappa=0$ for the zero-suction case, $U_{o}, V_{o}, W_{o}$ are velocity scales, and $L$ is the length scale in the flow-acceleration direction $z$. If the attachment line is assumed to be infinitely long, the velocities become functions of $z$ and $y$ only, and the similarity solution can be found.

The equations for the base flow were given by Hall et al. (1984). If the solutions of these equations are nondimensionalized with respect to the attachment-line velocity $U_{o}$, the boundary-layer thickness $\delta$, and the kinematic viscosity $\nu$, then the base flow is

$$
U(Y)=\hat{U}(Y), \quad V(Y)=\frac{1}{R} \hat{V}(Y), \quad \text { and } \quad W(Y, Z)=\frac{Z}{R} \hat{W}(Y)
$$


where $\{X, Y, Z\}=\{x, y, z\} / \delta$, the hats refer to similarity variables, and $\hat{U}, \hat{V}, \hat{W}$ are obtained by solving the equations described by Hall et al. (1984). Note that in the character of this similarity solution, $U$ and $V$ are uniform along the attachment line and $W$ varies linearly with distance from the attachment line. Because of the properties of this base flow, both temporal and spatial DNS approaches should yield equivalent results in the 2D limit for small-amplitude disturbances. However, the temporal DNS assumes that disturbances are growing in time and that there exists a linear transformation from temporal growth to the realistic spatially growing instabilities. Hall and Malik (1986) realized subcritically growing instabilities with a temporal DNS code, and, hence the difference between the weakly-nonlinear theory and the previous computations should not be attributable to the temporal DNS approximation. Although many previous studies have made use of the temporal approach because of the computational savings over the spatial formulation, the spatial and temporal formulations are only related in the linear limit, with the spatial formulation being more representative of the true physical problem.

The disturbance portion of equation (1) is found by solving the $3 \mathrm{D}$ incompressible Navier-Stokes equations in disturbance form as

$$
\frac{\partial \underline{u}}{\partial t}+(\underline{u} \cdot \nabla) \underline{u}+(\underline{U} \cdot \nabla) \underline{u}+(\underline{u} \cdot \nabla) \underline{U}=-\nabla p+\frac{1}{R} \nabla^{2} \underline{u}
$$

with the continuity equation and boundary conditions

$$
\underline{u}=0 \text { at } Y=0 \text { and } \quad \underline{u} \rightarrow 0 \quad \text { as } \quad Y \rightarrow \infty
$$

For this study, disturbances are forced by suction and blowing at the wall or as unsteady inflow conditions. At the inflow, solutions of the base flow (plus disturbances) are forced, and the buffer-domain technique by Streett and Macaraeg (1989) is employed as the outflow condition. 


\section{Form of Disturbances}

In general, disturbances on and near a 3D attachment-line region are of the 3D nature, requiring solutions of the full 3D Navier-Stokes equations. However, as assumed in the original theoretical study by Hall et al. (1984) and confirmed in the DNS computations by Spalart (1989) and Joslin (1995), a single mode in the attachment-line region of swept Hiemenz flow can take the form

$$
u=u(x, y, t), \quad v=v(x, y, t), \quad \text { and } \quad w=w(x, y, t) \times Z
$$

This form permits the $w$-velocity component of the disturbance to have a linear variation with distance from the attachment line, which is the same as the base flow (see eqn. 2). While the amplitude of $w$ varys linearly with distance from the attachment line, the $u$ and $v$ components remain uniform with distance from the attachment line. The subsequent computations by Jiménez et al. (1990) and Theofilis (1993a, 1993b) used this same disturbance form and showed linear results near the neutral curve which were in agreement with the Hall et al. (1984) theory and nonlinear results that failed to achieve the subcritical growth predicted by the weakly-nonlinear theoretical and computational results of Hall and Malik (1986).

In the present study, an alternate disturbance form is first used. Namely, the $w$ velocity component of the disturbance and the transverse shear of the mean flow are negligible; the disturbance becomes truely $2 \mathrm{D}$ along the attachment line. This implies that $w=0$ and $\partial w / \partial Z=0$ on the attachment-line. Although this simplification is not consistent with the equations of motion, it turns out that the neglected terms have little effect on the qualitative behavior of the computed disturbances. This assumption allows us to use a pre-existing DNS solver, which has been tested for 2D instabilities and 3D spanwise periodic disturbances in 2D and 3D base flows. This 2D assumption is arguably valid because the flow is overwhelming dominated by the flow in the attachmentline direction. The results will show that this disturbance assumption is apparently valid 
near the neutral curve and that the nonlinear subcritical trends agree both with the theory and with the disturbance form in eqn. (5); however, quantitative agreement is not achieved for the nonlinear comparisons.

A final series of simulations is performed with the linear variance form described by eqn. (5) and used by Hall and Malik (1986) for their theory and computations and used in subsequent computations by Jiménez et al. (1990) and Theofilis (1993a, 1993b). This dependence requires solutions of the following momentum and continuity equations.

$$
\begin{gathered}
\frac{\partial u}{\partial t}+(U+u) \frac{\partial u}{\partial X}+(V+v) \frac{\partial u}{\partial Y}+v \frac{\partial U}{\partial Y}=-\frac{\partial p}{\partial X}+\frac{1}{R}\left[\frac{\partial u^{2}}{\partial X^{2}}+\frac{\partial u^{2}}{\partial Y^{2}}\right] \\
\frac{\partial v}{\partial t}+(U+u) \frac{\partial v}{\partial X}+(V+v) \frac{\partial v}{\partial Y}+v \frac{\partial V}{\partial Y}=-\frac{\partial p}{\partial Y}+\frac{1}{R}\left[\frac{\partial v^{2}}{\partial X^{2}}+\frac{\partial v^{2}}{\partial Y^{2}}\right] \\
\frac{\partial w}{\partial t}+(U+u) \frac{\partial w}{\partial X}+(V+v) \frac{\partial w}{\partial Y}+v \frac{\partial W}{\partial Y}+(2 W+w) w=-\frac{\partial p}{\partial Z}+\frac{1}{R}\left[\frac{\partial w^{2}}{\partial X^{2}}+\frac{\partial w^{2}}{\partial Y^{2}}\right] \\
\frac{\partial u}{\partial X}+\frac{\partial v}{\partial Y}+w=0
\end{gathered}
$$

The results for the disturbance described by eqns. (6-9), hereafter referred to as 3D disturbances, will be shown to qualitatively agree with a 2D solution and the theory of Hall and Malik (1986) provided the disturbance pressure gradient is of a particular form in the flow-acceleration direction.

\section{Numerical methods of solution}

In the attachment-line $(X)$ direction, fourth-order central finite differences are used for the pressure equation and sixth-order compact differences are used for the momentum equations in the interior of the computational domain. At the boundary and near-boundary nodes, fourth-order forward and backward differences are used. The discretization yields a pentadiagonal system for the finite-difference scheme and a tridiagonal system for the compact-difference scheme. The approximations can be solved efficiently by appropriate backward and forward substitutions. 
In the wall-normal $(Y)$ direction, Chebyshev series are used to approximate the disturbances at Gauss-Lobatto collocation points. A Chebyshev series is used in the wallnormal direction because it provides good resolution in the high-gradient regions near the boundaries. Furthermore, the use of as few grid points as possible results in significant computational cost savings. In particular, the use of the Chebyshev series enables an efficient pressure solver. Because this series and its associated spectral operators are defined on $[-1,1]$ and the physical problem of interest has a truncated domain $\left[0, y_{\max }\right]$, a transformation is employed. Furthermore, a stretching function is used to cluster the grid near the wall. For further details on the properties and the use of spectral methods, refer to Canuto et al. (1988).

For time marching, a time-splitting procedure was used with implicit Crank-Nicolson differencing for normal diffusion terms; an explicit three-stage Runge-Kutta (RK) method by Williamson (1980) was used for the remaining terms. For details of the time-marching procedure, refer to Joslin et al. (1992). The intermediate RK velocities are determined semi-implicitly, the pressure is found by solving the Poisson equation, and the full RK stage velocities are obtained by correcting the intermediate velocities with the updated pressure. The above system is solved three consecutive times to obtain full time-step velocities.

To satisfy global mass conservation, an influence-matrix method is employed and is described in some detail by Streett and Hussaini (1991), Danabasoglu et al. (1990, 1991), and Joslin et al. (1992). For boundary-layer flow, four Poisson-Dirichlet problems are solved for the discrete mode that corresponds to the zero eigenvalue of the system; single Poisson-Neumann problems are solved for all other modes. To efficiently solve the resulting Poisson problem, the tensor-product method of Lynch et al. (1964) is used.

The buffer-domain technique introduced by Streett and Macaraeg (1989) is used for the outflow condition. As shown by Joslin et al. (1992) for the flat-plate boundary-layer problem, a buffer length of three disturbance wavelengths is adequate for traveling waves. 
The disturbances are assumed to be from the discrete spectrum, which exponentially decay with distance from the wall. Both at the wall and in the far field, homogeneous Dirichlet conditions are imposed. The base flow is used for the inflow boundary condition.

Finally, disturbances are forced as unsteady inflow conditions or by unsteady suction and blowing of the wall-normal velocity component through the wall. For the former forcing, $u$ and $v$ profiles that are normalized by $u$ are generated by some theory (e.g., quasi-parallel linear stability theory), and an amplitude is imposed. For the later forcing, a harmonic source is introduced, the amplitude is based on the wall-normal velocity, and the wave profiles develop naturally in the flow. A similar technique has been used by (among others) Danabasoglu et al. (1991) in their study of flow control by suction and blowing in a channel flow. Although the disturbances may be generated by random frequency input, the disturbances of interest here are forced with known frequencies. Essentially, this disturbance generator is an alteration to the no-slip boundary conditions, which are conventionally used for the wall condition in a viscous flow problem.

\section{Linear Stability of Swept Hiemenz Flow}

Here, an assessment is made in regard to the value of the Orr-Sommerfeld/Squire equations (OS) formulation in attachment-line flow. Note that OS involves a quasi-parallel flow assumption (i.e., $V=0$ ), and that no amplitude information is included in the theory. Figure 3 shows the neutral curves predicted with both the OS solver and the linear theory of Hall et al. (1984), which accounts for all linear terms (i.e., nonparallel theory). The nonparallel theory allows for a developing boundary layer (i.e., $V \neq 0$ ). The largest disagreement in these results appears near the critical-point region. The theory of Hall et al. (1984) predicts a critical Reynolds number that is more consistent with the experiments, as shown in figure 2. Although accurate growth rates of disturbances may not be obtained with OS as a result of the quasi-parallel constraint, a good estimate of instability wavelengths can be obtained. For example, with the Reynolds number $R=800$ and the 
frequency $\omega=0.1271$, Hall et al. (1984) listed the wave number $\alpha_{r}=0.3385$. According to OS for the same Reynolds number and frequency, the wavenumber $\alpha_{r}=0.3382$ is obtained. Therefore, the terms that are neglected in the governing OS equations, but retained in the Hall et al. (1984) theory, primarily affect the growth and decay rates of the instabilities. Obviously, the spatial growth of the disturbances are of primary importance in transition studies; however, the OS tool can be used to quickly generate base disturbance quantities such as $\alpha_{r}=f(R, \omega)$ and profiles. These quantities can be used, for example, to determine the initial states for simulations. Although beyond the scope of the present study, this comparison indicates the accuracy of OS in predicting attachment-line instabilities and demonstrates how the nonparallel theory of Hall et al. (1984) improved upon conventional OS.

Figure 3 also shows the locations on the Reynolds number/frequency plane where the DNS is used to study the linear and nonlinear instabilities for the attachment-line flow. The simulations are performed on a grid of 661 points ( $\simeq 60$ points per wavelength) along the attachment line and 81 points in the wall-normal direction. The far-field boundary is located at $50 \delta$ from the wall, and the computational length along the attachment line is $216.56 \delta$. This attachment-line length corresponds to 11 wavelengths for $R=570$ and $\omega=0.1249$. For the time-marching scheme, the disturbance wavelength was divided into 320 time steps per period for small-amplitude disturbances and into 2560 time steps for large-amplitude disturbances (stability considerations). The total Cray Y-MP time for a simulation with a single processor was $1.5 \mathrm{hrs}$ for small-amplitude disturbances and 12.0 hrs for large-amplitude disturbances.

Disturbances for the first simulations are forced at the computational inflow with an amplitude of $A=0.001 \%$ (i.e., arbitrary small amplitude). The Reynolds number $R=570$ and the frequency $\omega=0.1249$ correspond to the region of subcritical growth found by Hall and Malik (1986), where disturbances are linearly stable. Disturbances that evolve in both 
a base flow that complements the quasi-parallel OS assumptions $(V=0)$ and the full, swept Hiemenz flow are computed with DNS. Although not shown here, the computed disturbance decay rate and the wavelength in the quasi-parallel flow agree exactly with OS. The disturbance that propagates in the complete swept Hiemenz flow closely retains the wavelength predicted by OS, but decays at a slower rate than that predicted by OS. This change in decay rate is consistent with the theory of Hall et al. (1984). From this comparison, we find that the wall-normal velocity $(V)$ terms in the stability equations have a destabilizing effect on the disturbance, which results in the modified neutral curve shown in figure 3.

Additional simulations were conducted in the regions near branches I and II and in the critical Reynolds number region to confirm the neutral curve predicted by the theory. The growth and decay of various frequency waves compared with the neutral solutions were in agreement with the neutral curve as predicted theoretically by Hall et al. (1984), computed by Spalart (1989), and computed more recently by Jiménez et al. (1990) and Theofilis (1993a, 1993b). This suggests that the chordwise strain contribution, which was neglected from the 2D DNS solver is insignificant for linear computations near the neutral curve.

Finally, the effect of both steady suction and steady blowing on linear instability growth in this region was documented. The results indicate that suction stabilizes the disturbance and blowing significantly destabilizes the disturbance. The effects of suction and blowing on disturbances computed by DNS are in agreement with the theory of Hall et al. (1984) for small-amplitude disturbances.

\section{Nonlinear Growth of Subcritical Instabilities}

Although the theoretical and computational results agree for the growth and decay properties of linear disturbances along the attachment line, the nonlinear results differ in the subcritical behavior of disturbances. To resolve this discrepancy, the computed results 
from the present study are compared with the previous studies of Hall and Malik (1986), Jiménez et al. (1990), and Theofilis (1993b). In addition, the effects of suction on unstable modes are documented.

Figure 4 shows the evolution of the fundamental wave, the mean-flow distortion, and the harmonics from a simulation forced at the inflow with a large amplitude of $A=$ $12 \%$, a Reynolds number of $R=570$, and a frequency of $\omega=0.1249$. After a transient region of adjustment, the fundamental wave encounters subcritical growth, which is in agreement with the weakly nonlinear theory. Instantaneous and mean streamwise and wall-normal velocity profiles at various attachment-line locations are shown in figures 5 and 6 , respectively. The results in figure 5 indicate that time-dependent distortions to the base flow are observed, but the mean flow $\left(U+u_{o}\right)$, which consists of the base flow and the mean-flow distortion components, shows no noticable deviation from the base-flow solution. However, the results in figure 6 indicate that both the time-dependent and mean wall-normal profiles undergo distortions because of the disturbance. To help understand what effect these mean distortions would have on linear stability calculations, figure 7 shows the wall-normal component of the base flow that corresponds to $R=570$ and $R=670$. A comparison of these base-flow profiles with the mean flow of figure 6 shows that a large-amplitude disturbance produces a distortion to the base flow, which causes an effective increase in the base Reynolds number. Although the increase in Reynolds number alone does not account for the growing mode (based on linear stability analysis with the same frequency), we surmise that (similar to nonparallel effects) nonlinear disturbances broaden the neutral curve toward higher frequencies and lower critical Reynolds numbers. However, these lower Reynolds numbers are no where near the $R_{\theta}=100$ limit for bypass transition in experiments.

To control the subcritical growth of disturbances, various levels of suction are employed. Although Hall and Malik (1986) noted that suction makes the flow more suscepti- 
ble to subcritical instability growth, figure 8 shows that this subcritical disturbance growth can be controlled by using small levels of suction. If the 2D DNS results mimic the actual 3D behavior of the flow, then large-amplitude disturbances generated on the attachment line can be controlled with a sufficient amount of suction.

To determine if nonlinear disturbance growth can be found above branch II of the neutral stability curve and to ensure that the subcritical growth obtained both by Hall and Malik (1986) and by DNS shown in figure 4 did not artificially result from the disturbance forcing at the inflow boundary, the next sequence of simulations are forced by suction and blowing at decaying modes that correspond to $R=684.2$ and $\omega=0.1249$ and are repeated at $R=684.2$ and $\omega=0.1230$ (closer to branch II of the neutral curve). For the later test point, the initial amplitudes of the disturbances for each simulation were incrementally increased; the resulting disturbance evolutions are shown in figure 9 (normalized by the initial amplitude to show the relative growth effects). The otherwise linearly decaying mode becomes amplified because of the nonlinear forcing. Interestingly, as the initial amplitude is increased, the fundamental wave receives a smaller percentage of the total energy injected into the flow. The evolution of the largest-amplitude disturbance and the effects of steady suction on the disturbance amplitude are shown in figure 10. The results further demonstrate that small amounts of suction can be used to stabilize disturbances that otherwise nonlinearly grow near branch II of the neutral curve. Larger forcing amplitudes are required to obtain nonlinear growth with Reynolds numbers and frequencies further away from the neutral curve, and, as expected, larger amounts of suction are required to stabilize the disturbances.

At this point it is not clear why the results of Jiménez et al. (1990) do not agree with either the present DNS results or the previous theory and computations of Hall and Malik (1986); however, from the present initial amplitudes required to achieve this subcritical growth, Theofilis (1993b) apparently could not force a disturbance with sufficient amplitude 
to realize this nonlinear growth.

\section{3D Nonlinear Subcritical Instabilities}

It should be noted that the present results are achieved through the $2 \mathrm{D}$ simplification. In this section, the 3D instabilities are determined by solving eqns. (6)-(9). Note, that by using the disturbance form given in eqn. (5), the $Z$ dependence of the disturbance is removed from the theoretical/computational problem, except for a partial derivative of the pressure in eqn. (8). In fact, it is from this observation that we find a difference between the studies of Hall and Malik (1986) and Jiménez et al. (1990). It is apparent from the manuscripts that different assumptions were made for the pressure behavior in the flow-acceleration direction.

In the studies of Hall et al. (1984) and Hall and Malik (1986), the disturbance pressure was a function of $(X, Y)$ only. This leads to

$$
\frac{\partial p}{\partial Z}=0 \times Z
$$

in eqn. (8) above. With this pressure form, a series of simulations were conducted by solving eqns. (6)-(9). Figure 11 shows the fundamental mode and first harmonic of the attachment-line direction velocity component compared with the previous 2D mode (Fig. 4). In agreement with the $2 \mathrm{D}$ qualitative behavior, the $3 \mathrm{D}$ mode undergoes subcritical growth. Quantitative differences are apparent and expected due to the addition of eqn. (8) and the modified continuity equation. The energy content with distance along the attachment line is probably a better measure of total disturbance growth or decay. Figure 12 shows this disturbance energy for various subcritical Reynolds numbers. For a fixed initial disturbance amplitude, it is clear that the disturbance energy increases with distance along the attachment line, in agreement with the theory and computations of Hall and Malik (1986) and with the earlier 2D modal approximation.

In the study of Jiménez et al. (1990), the disturbance pressure was assumed to be of the same form of the base flow. Namely, pressure varied with the square of distance from 
the attachment-line in the flow-acceleration direction. They arrived at a pressure gradient in the flow-acceleration direction which took the form

$$
\frac{\partial p}{\partial Z}=-1 \times Z
$$

Using this pressure form, a final simulation was conducted and the results are compared in Figure 13 with the results using eqn. (10) as the flow-acceleration pressure gradient. This simple difference in pressure leads to a decaying mode instead of nonlinear subcritical growth. Hence, the disrepancy between the Jiménez et al. (1990) computations and the computations and weakly-nonlinear theory of Hall and Malik (1986) lie with an effective pressure source difference.

This leads to an additional puzzling question: which pressure form should be used for future simulations? To address this question, we turn to the fully 3D simulations of Joslin (1995) for guidance. In those simulations, no aprior assumption was made for flowacceleration disturbance behavior. A harmonic-source suction and blowing disturbance generator initiated the disturbances at a given frequency. Joslin (1995) showed that the $w$ disturbance velocity varied linearly with distance from the attachment line, in agreement with the form described by eqn. (5). However, no attempt was made to study the disturbance pressure field. At an arbitrary distance downstream of the source, the database of Joslin (1995) was explored for pressure behavior and is shown in Figure 14, where the maximum pressure is shown with distance from the attachment line. The results clearly show that the pressure is uniform with distance from the attachment line for this type of disturbance. This uniformity supports the pressure form of Hall and Malik (1986).

\section{Concluding remarks}

In this study, results are presented for the spatial direct numerical simulations (DNS) of the $2 \mathrm{D}$ and $3 \mathrm{D}$ disturbances that propagate along the attachment line of a swept Hiemenz flow. With a quasi-parallel base-flow approximation, the small-amplitude disturbances 
were shown to grow and decay in agreement with linear stability theory. The true swept Hiemenz base flow leads to a destabilization of disturbances, which agrees with the nonparallel theory of Hall et al. (1984). Furthermore, the effect of steady suction and blowing on small-amplitude disturbances was documented with DNS. In agreement with the results of Hall et al. (1984), suction stabilizes and blowing destabilizes the small-amplitude disturbances.

Subcritical nonlinear disturbance growth was detected with a weakly-nonlinear theory and computations by Hall and Malik (1986). Later, DNS studies by Theofilis (1993b) and Jiménez et al. (1990) failed to find this nonlinear disturbance growth. The present 2D and 3D simulations have detected nonlinear subcritical disturbance growth; these results support the former theoretical and computational results of Hall and Malik (1986). Based on the present results, the computations by Theofilis (1993b) may not have achieved subcritical growth because the forcing amplitudes were apparently too small. Furthermore, Jiménez et al. (1990) apparently used a different disturbance pressure form in the flowacceleration direction. The present study showed that this assumed variation in pressure leads to a decaying subcritical mode, which qualitatively agrees with the results of Jiménez et al. (1990). These results suggest that the reason for the discrepancy may evidently be attributable to differing disturbance pressure forms. The 3D DNS data of Joslin (1995) tends to support the pressure form used by Hall and Malik (1986) for the types of disturbances considered.

Furthermore, the DNS results demonstrate that steady suction stabilizes the otherwise nonlinearly growing disturbances. No nonlinear growing disturbances were detected near branch I of the neutral curve; however, equilibrium-like states were found near branch I.

Although the present study has served to dissolve the previous discrepancy surrounding the subcritical growing disturbances, the results have not explained the physics of the flow between the known limit of linear instability $R_{\theta} \simeq 245$ and the bypass (or turbulence) 
limit of $R_{\theta} \simeq 100$. The present nonlinear results suggest that the linear critical Reynolds can be slightly reduced due to nonlinear effects; however, the true swept-wing bypass problem likely involves potentially large and multi-frequency/multi-wavenumber $3 \mathrm{D}$ disturbances. Hence, the explanation for bypass transition will involve these multiple modes, which may be generated off the attachment line. Furthermore, Joslin (1995) has shown with fully $3 \mathrm{D}$ simulations that disturbance packets generated off but near the attachment line can transfer energy to the attachment-line region.

Hall \& Seddougui (1990) studied oblique waves and their interaction in attachmentline flow at the large Reynolds number limit. They note that close to the attachment line a small band of destabilized oblique modes appear, interact with the $2 \mathrm{D}$ mode, and cause a breakdown of the $2 \mathrm{D}$ mode. In addition, they note that oblique modes become less important away from the attachment line and that low-frequency modes become the dominant mechanism (i.e., stationary crossflow modes). Lin \& Malik (1994) performed 3D linear computations which showed that, in addition to the dominant $2 \mathrm{D}$ symmetric wave (studied here), both asymmetric and symmetric modes, which have phase differences with distance from the attachment line, can be unstable depending on the Reynolds number. Although these new modes are linearly stable in the subcritical region outlined by Hall and Malik (1986), perhaps some combination of small (but finite) amplitude modes may lead to a better understanding of the region between the linear and bypass Reynolds numbers.

\section{References}

Arnal, D. (1994) Boundary layer transition: prediction, application to drag reduction. AGARD FDP/VKI course on Skin friction drag reduction, Brussels, Belgium/March 2-6, 1992.

Canuto, C., Hussaini, M. Y., Quarteroni, A. and Zang, T. A. (1988) Spectral Methods in Fluid Dynamics. Springer, New York. 
Cumpsty, N. A. and Head, M. R. (1969) The calculation of the three-dimensional turbulent boundary layer. Part III. Comparison of attachment-line calculations with experiment. Aeron. Quart. 20,99-113.

Danabasoglu, G., Biringen, S. and Streett, C. L. (1990) Numerical simulation of spatially-evolving instability control in plane channel flow. AIA A Paper No. 90-1530.

Danabasoglu, G., Bringen, S. and Streett, C. L. (1991) Spatial simulation of instability control by periodic suction and blowing. Phys. Fluids A, 3(9), 2138-2147.

Gad-el-Hak, M. and Bushnell, D. M. (1991) Separation control: Review. J. Fluids Engng., 113, 5-30.

Gaster, M. (1965) A simple device for preventing turbulent contamination on swept leading edges. J. Roy. Aero. Soc. 69, 788-789.

Gaster, M. (1967) On the flow along swept leading edges. Aeron. Quarterly 18, $165-184$.

Gregory, N. and Love, E. M. (1965) Laminar flow on a swept leading edge. Final Progress Report, NPL Aero. Memo., 26.

Hall, P., Malik, M. R. and Poll, D. I. A. (1984) On the stability of an infinite swept attachment line boundary layer. Proc. Roy. Soc. Lond. A A395, 229-245.

Hall, P. and Malik, M. R. (1986) On the instability of a three-dimensional attachmentline boundary layer: Weakly nonlinear theory and a numerical simulation. J. Fluid Mech. 163, 257-282.

Hall, P. and Seddougui, S. O. (1990) Wave interactions in a three-dimensional attachment-line boundary layer. J. Fluid Mech. 217, 367-390.

Hiemenz, K. (1911) Die grenzschicht an einem in den gleichförmigen flüssigkeitsstrom eingetauchten geraden kreiszylinder. Thesis, Göttingen 1911, Dingl. Polytechn. J., 326, 
321.

Jiménez, J., Martel, C., Agüí, J. C. and Zufiria, J. A., (1990) Direct numerical simulation of transition in the incompressible leading edge boundary layer. ETSIA/MF-903.

Joslin, R. D., Streett, C. L. and Chang, C.-L. (1992) Validation of three-dimensional incompressible spatial direct numerical simulation code - a comparison with linear stability and parabolic stability equations theories for boundary-layer transition on a flat plate. NASA TP-3205.

Joslin, R. D. (1995) Direct simulation of evolution and control of three-dimensional instabilities in attachment-line boundary layers. J. Fluid Mech. 291, 369-392.

Lin, R.-S. and Malik, M.R. (1994) The stability of incompressible attachment-line boundary layers-A 2D-eigenvalue approach, AIAA Paper No. 94-2372.

Körner, H., Horstmann, K. H., Köster, H., Quast, A. and Redeker, G. (1987) Laminarization of transport aircraft wings: A German view. AIAA Paper No. 87-0085.

Lynch, R. E., Rice, J. R. and Thomas, D. H. (1964) Direct solution of partial difference equations by tensor product methods. Num. Math. 6, 185-199.

Maddalon, D. V., Collier, F. S., Jr., Montoya, L. C. and Putnam, R. J. (1990) Transition flight experiments on a swept wing with suction. Laminar-Turbulent Transition, (D. Arnal and R. Michel, eds.), Springer, Berlin, 53-62.

Pfenninger, W. (1965) Flow phenomena at the leading edge of swept wings. Recent Developments in Boundary Layer Research, AGARDograph 97, May 1965.

Pfenninger, W. and Bacon, J. W., Jr. (1969) Amplified laminar boundary-layer oscillations and transition at the front attachment line of a 45 degree swept flat-nosed wing with and without boundary-layer suction. Viscous Drag Reduction, (C. S. Wells, ed.), Plenum, 85-105. 
Poll, D. I. A. (1979) Transition in the infinite swept attachment line boundary layer. Aeron. Quarterly 30,607-628.

Poll, D. I. A. (1980) Three-dimensional boundary layer transition via the mechanisms of attachment-line contamination and crossflow stability. Laminar-Turbulent Transition, (R. Eppler and H. Fasel, eds.), Springer, Stuttgart, 253-262.

Poll, D. I. A. (1985) Some observations of the transition process on the windward face of a long yawed cylinder. J. Fluid Mech. 150, 329-356.

Reed, H. L. and Saric, W. S. (1986) Stability and transition of three-dimensional flows. In10th U.S. National Congress of Applied Mechanics, (J. P. Lamb, ed.), 457-468.

Spalart, P. R. (1989) Direct numerical study of leading-edge contamination. AGARDCP-438, 5.1-5.13.

Streett, C. L. and Hussaini, M. Y. (1991) A numerical simulation of the appearance of chaos in finite-length Taylor-Couette flow. Appl. Numer. Math. 7, 41-71.

Streett, C. L. and Macaraeg, M. G. (1989) Spectral multi-domain for large-scale fluid dynamic simulations. Int. J. Appl. Numer. Math., 6, 123-140.

Theofilis, V. (1993a) Numerical experiments on the stability of leading edge boundary layer flow: A two-dimensional linear study. Int. J. Num. Methods in Fluids 16, 153-170.

Theofilis, V. (1993b) A spectral velocity-vorticity algorithm for the solution of the incompressible Navier-Stokes equations. Numer. Methods in Laminar-Turbulent Flow, (C. Taylor, ed.), Pineridge, 801-811.

Tuttle, M. H. and Maddalon, D. V. (1982) Laminar flow control (1976-1982). NASA TM 84496.

Williamson, J. H. (1980) Low-storage Runge-Kutta schemes. J. Comput. Phys. 35(1), $48-56$. 


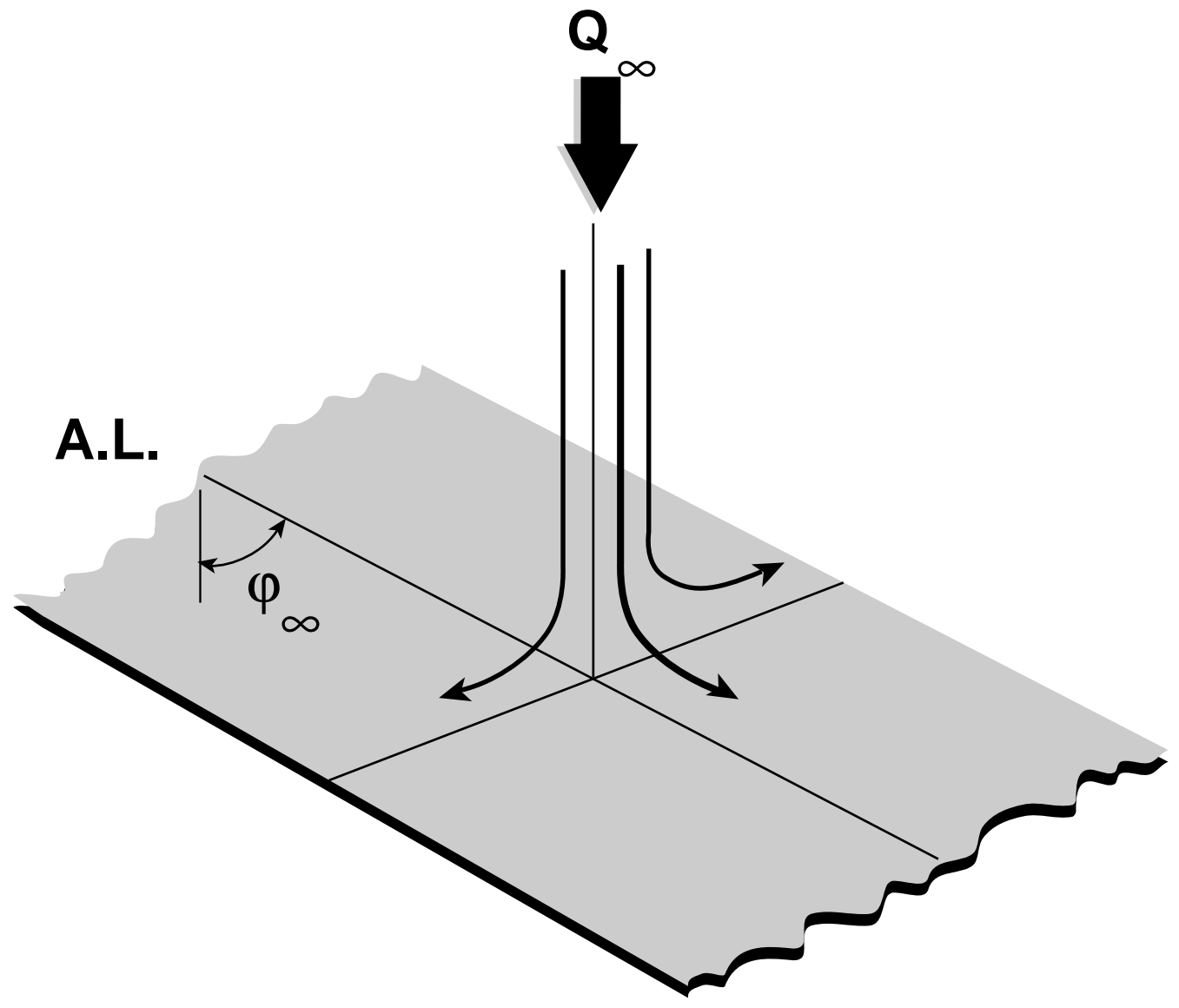

Figure 1. Sketch of attachment-line region of swept Hiemenz flow. 


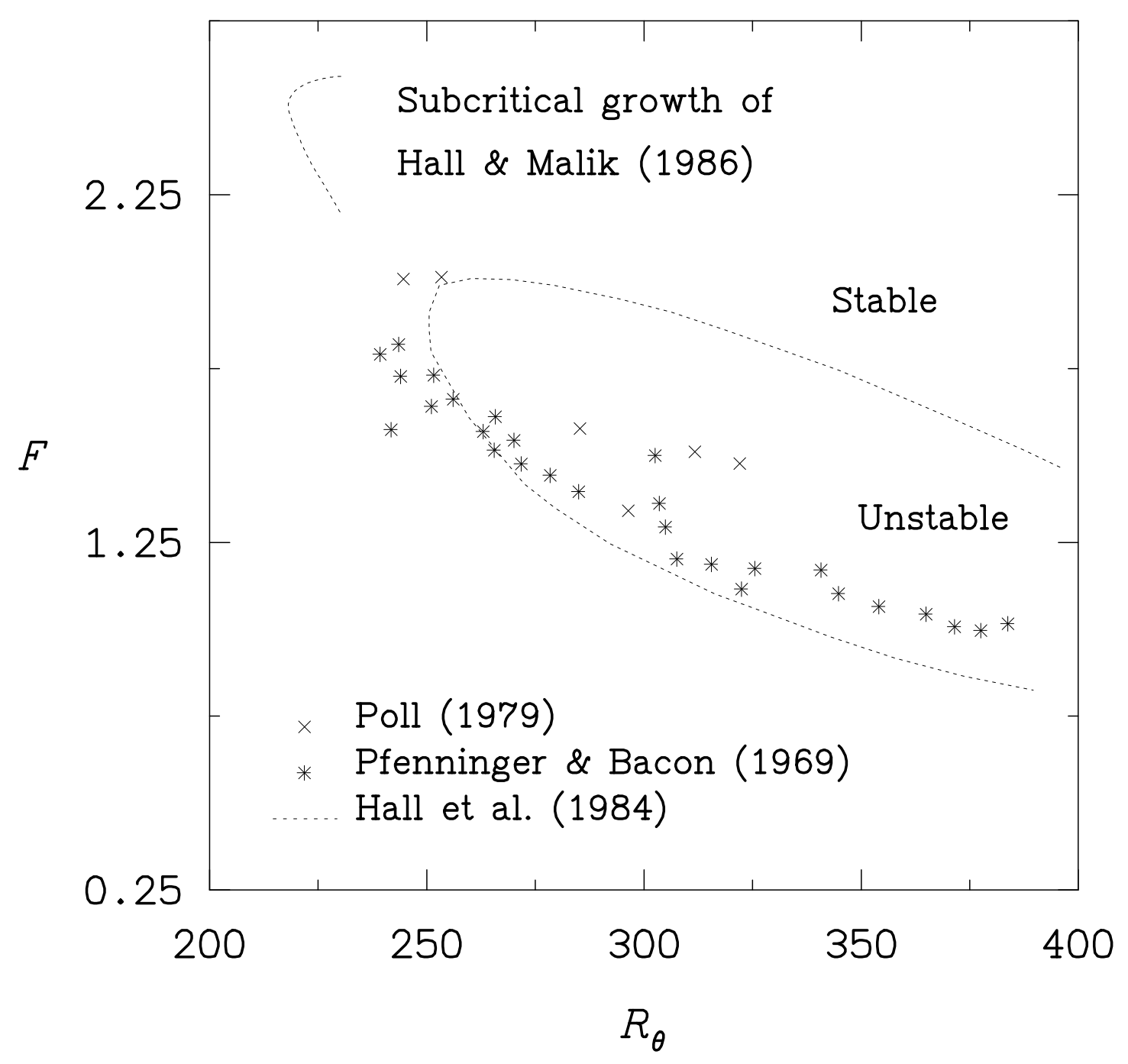

Figure 2. Neutral curve, experimental regions of instability growth, and theoretical region of subcritical growth in attachment-line boundary layer. (F-frequency and R-Reynolds number) 


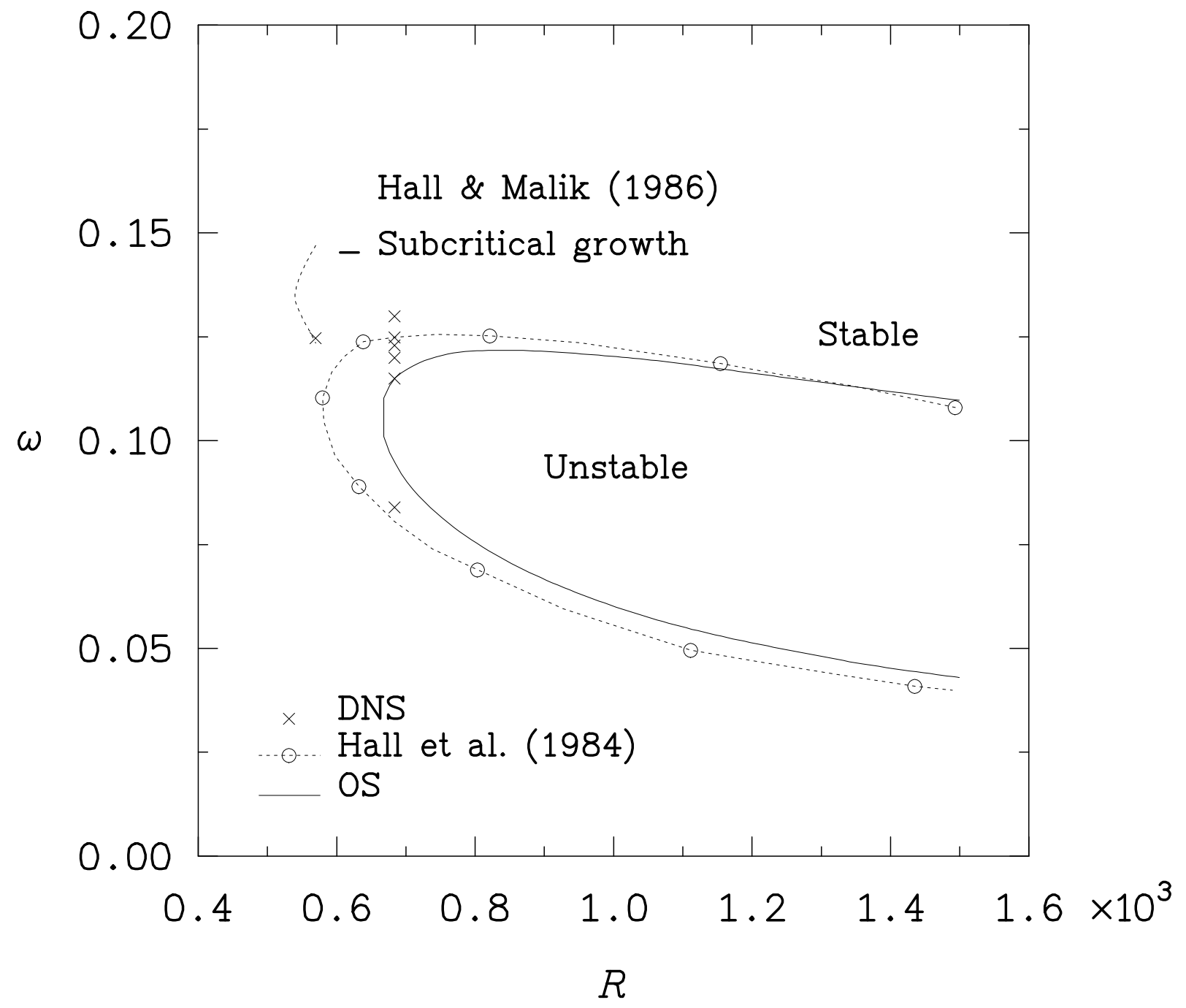

Figure 3. Neutral curves, region of subcritical instability growth, and sample points for DNS in attachment-line boundary layer. 


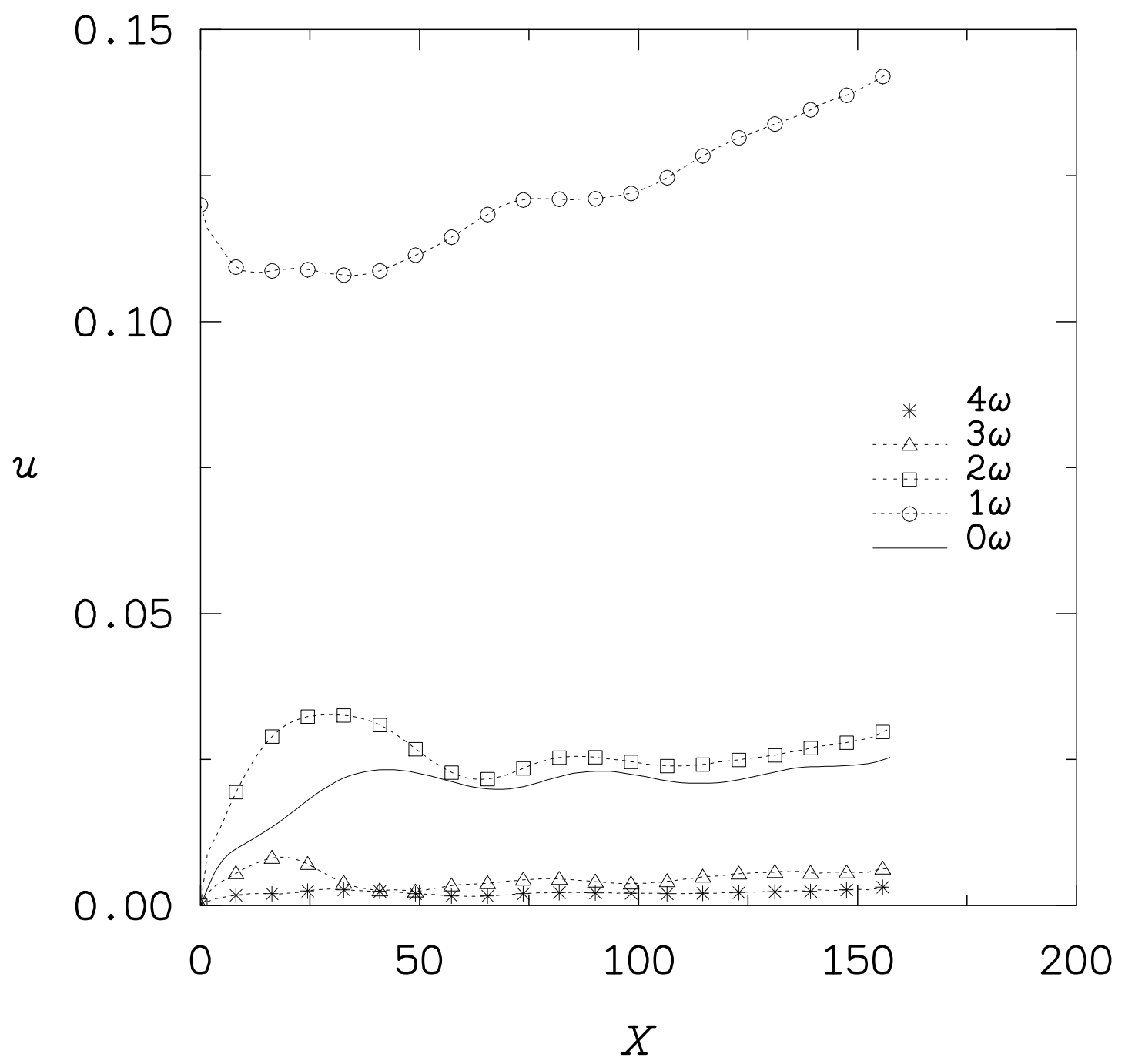

Figure 4. Nonlinear subcritical disturbance growth in attachment-line boundary layer at $R=570$ and $\omega=0.1249$. 

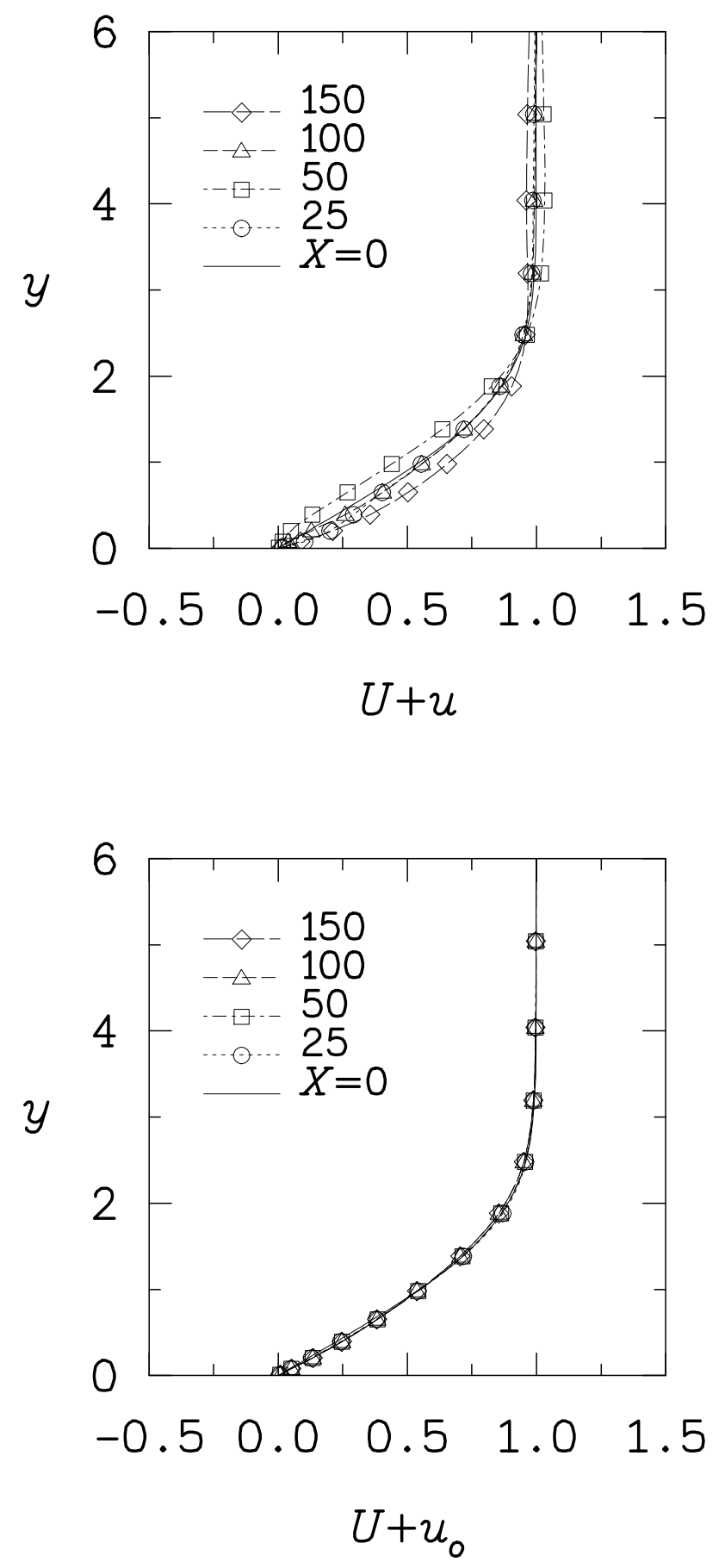

Figure 5. Instantaneous (top) and mean (bottom) streamwise velocity profiles of nonlinear, subcritically growing disturbance in attachment-line boundary layer at $R=570$ and $\omega=$ 0.1249 . 

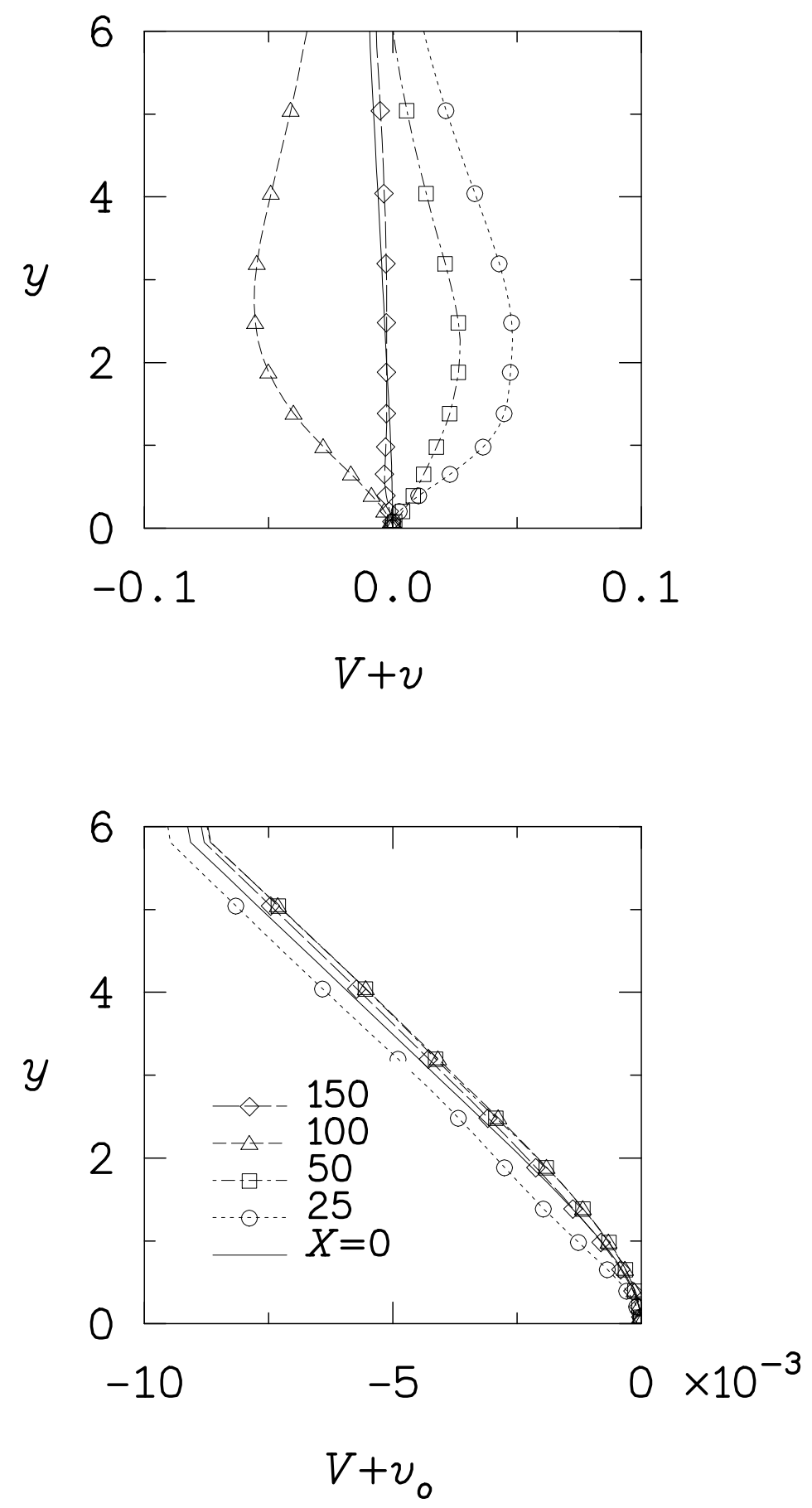

Figure 6. Instantaneous (top) and mean (bottom) wall-normal velocity profiles of nonlinear, subcritically growing disturbance in attachment-line boundary layer at $R=570$ and $\omega=0.1249$. 


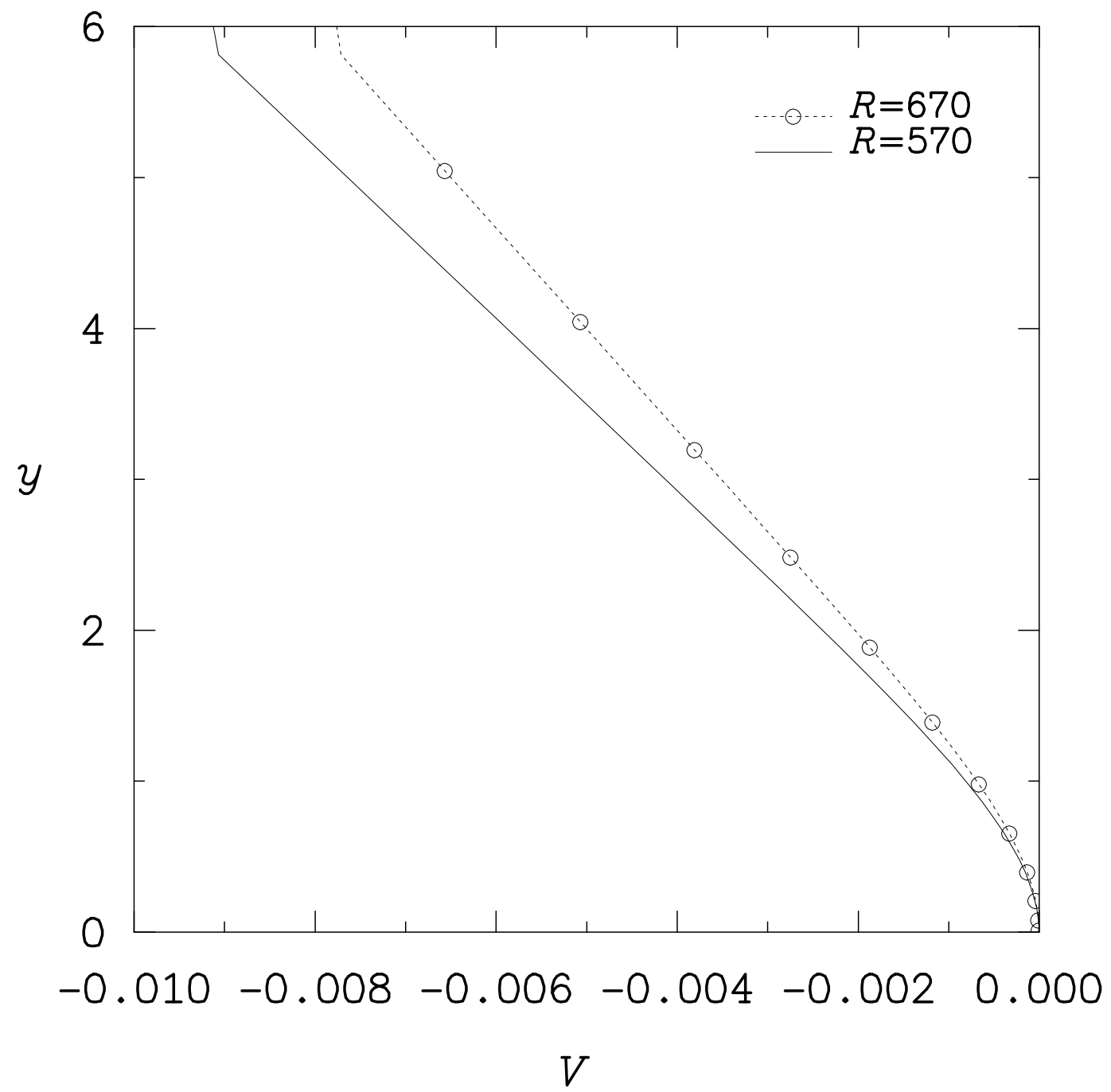

Figure 7. Wall-normal component of base flows that corresponds to $R=570$ and $R=670$. 


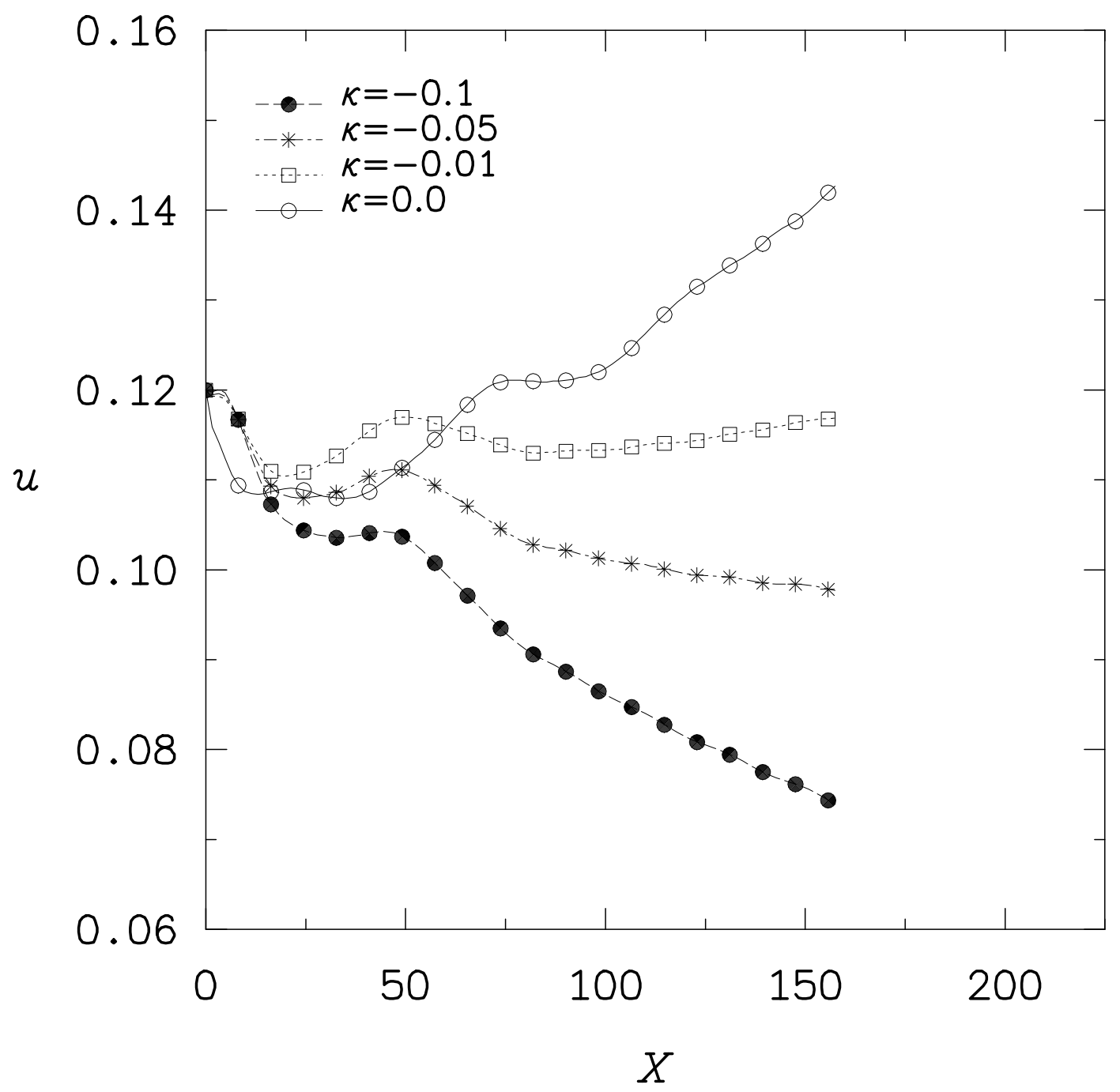

Figure 8. Control of nonlinear subcritical disturbance growth in attachment-line boundary layer at $R=570$ and $\omega=0.1249$ with suction. 


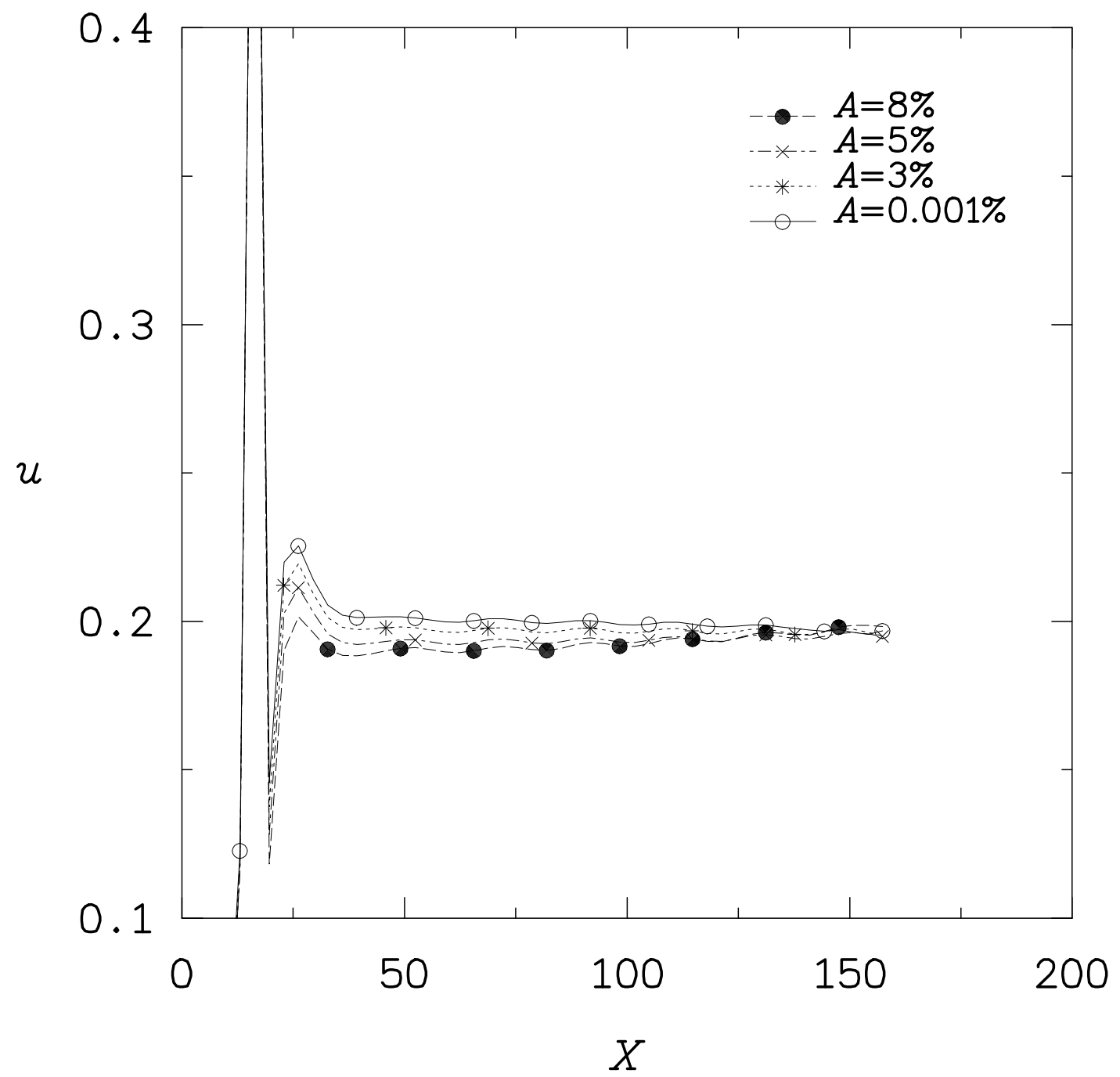

Figure 9. Nonlinear disturbance growth in attachment-line boundary layer at $R=684.2$ and $\omega=0.1230$. (Disturbances normalized by initial amplitudes.) 


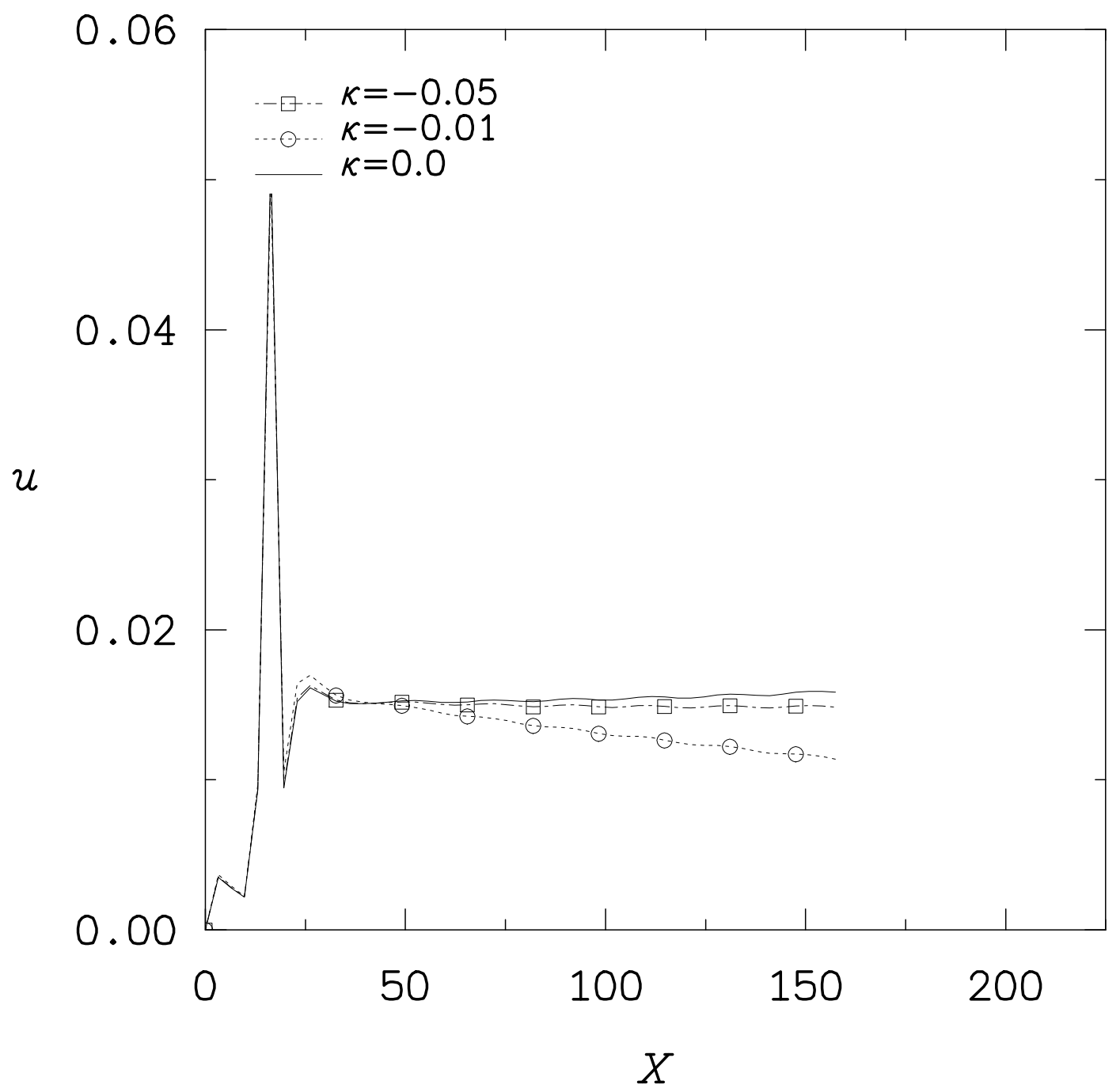

Figure 10. Control of nonlinear disturbance growth in attachment-line boundary layer at $R=684.2$ and $\omega=0.1230$ with suction. 


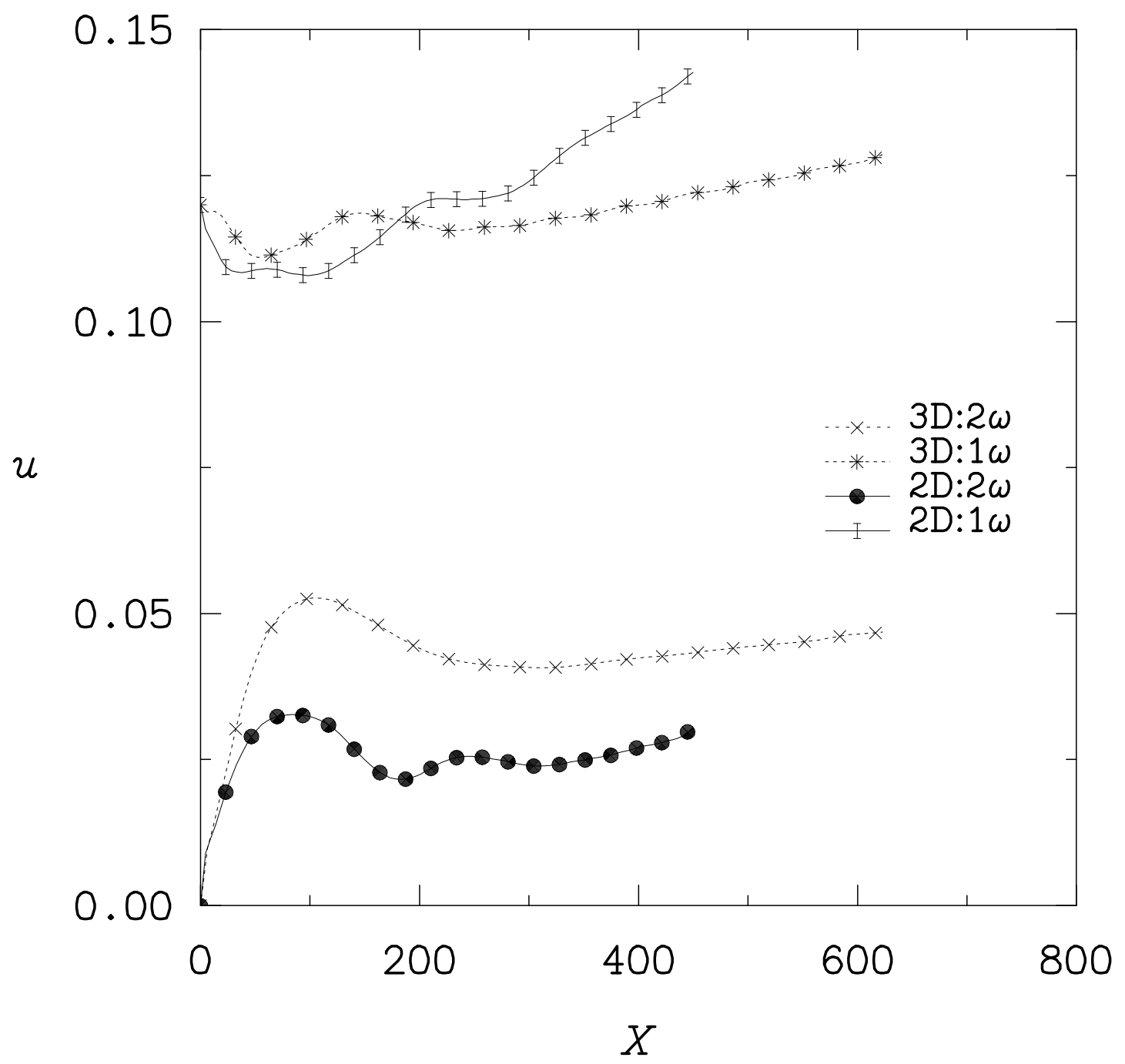

Figure 11. Nonlinear subcritical growth of $2 \mathrm{D}$ and $3 \mathrm{D}$ disturbances in attachment-line boundary layer at $R=570$ and $\omega=0.1249$. 


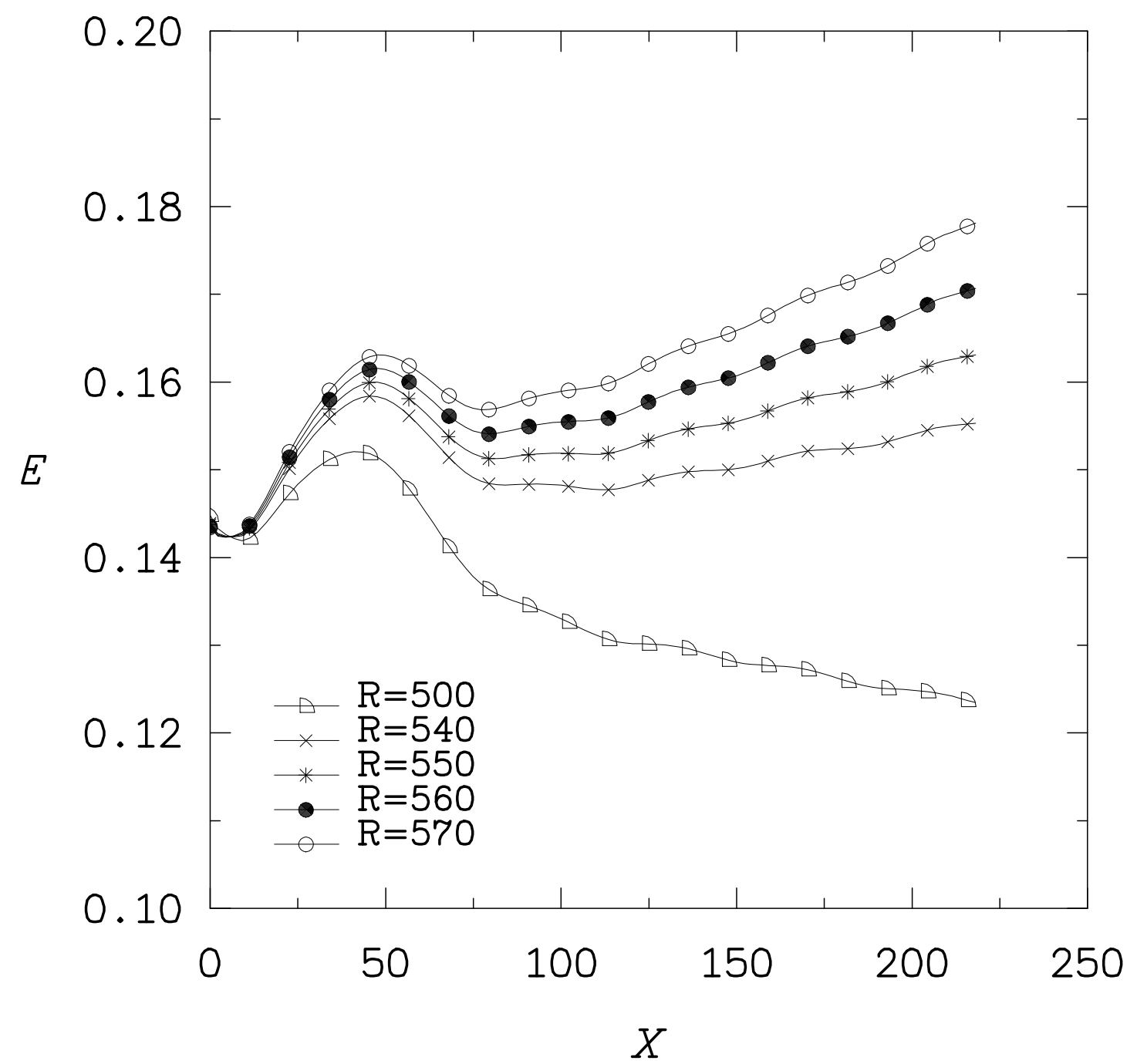

Figure 12. Nonlinear subcritical energy of 3D disturbances in attachment-line boundary layer with Reynolds number at $\omega=0.1249$. 


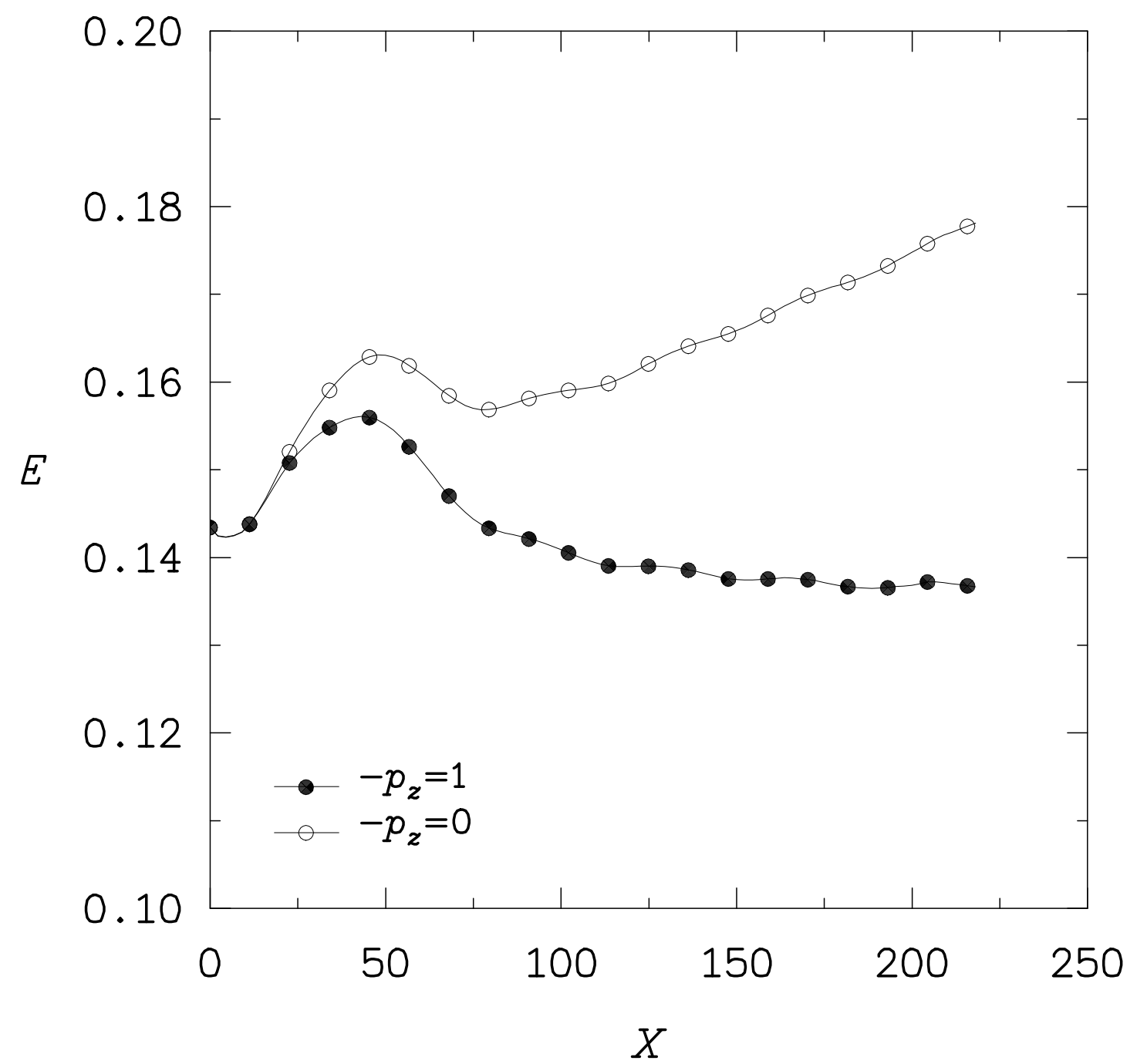

Figure 13. Nonlinear subcritical energy of 3D disturbances in attachment-line boundary layer at $R=570$ and $\omega=0.1249$. (Pressure gradients of $-\circ-$, Hall and Malik (1986) and -•-, Jiménez et al. (1990). 


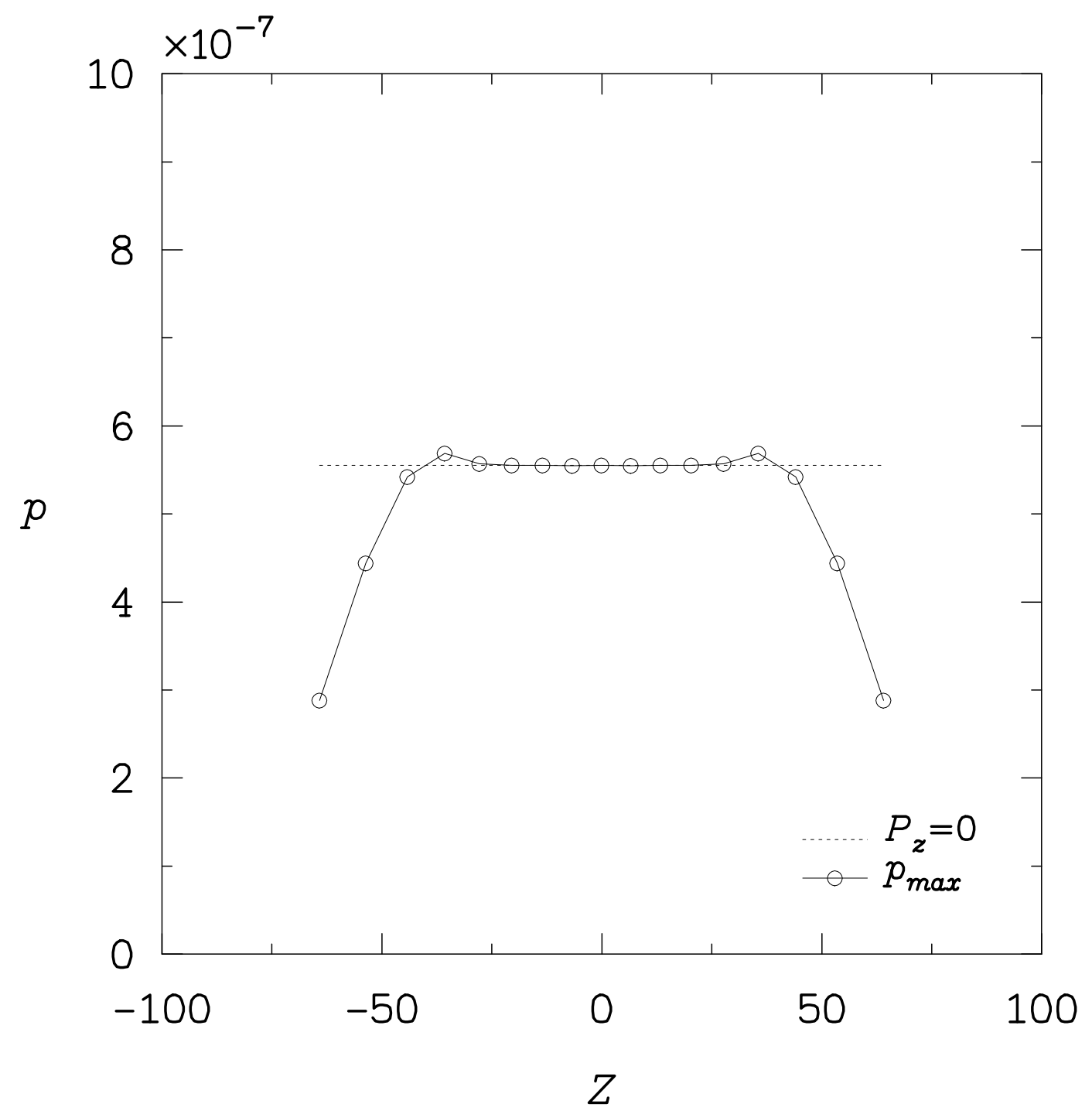

Figure 14. Maximum pressure with flow-acceleration direction at $X=100$ in attachmentline boundary layer with Reynolds number at $\omega=0.1249$. (Results of 3D simulation by Joslin (1995).) 